\title{
Polypharmacy among Older Advanced Lung Cancer Patients taking EGFR Tyrosine Kinase Inhibitors
}

\author{
Taiki Hakozaki ${ }^{1}$, Takuma Matsuo ${ }^{1}$, Akihiro Shimizu ${ }^{1}$, Yoko Ishihara ${ }^{1}$, and Yukio Hosomi ${ }^{1}$ \\ ${ }^{1}$ Tokyo Metropolitan Cancer and Infectious Diseases Center Komagome Hospital
}

July 2, 2020

\begin{abstract}
Aim: Polypharmacy (PP) is a common problem among the older adults and has a potential effect on health-related problems. However, the significance of PP in older advanced non-small cell lung cancer (NSCLC) patients and those on oral moleculartargeted anticancer agents is unclear. Methods: This retrospective study reviewed the records of 334 advanced NSCLC patients who underwent epidermal growth factor receptor tyrosine kinase inhibitor (EGFR-TKI) treatment. PP was defined as $>=5$ concomitant medications. Potentially inappropriate medication (PIM) use was measured using the updated screening tool of older people's prescriptions (STOPP) ver. 2 criteria. We also estimated survival distributions using the Kaplan-Meier method, compared between-group differences using the log-rank test, explored potential predictors of survival using Cox regression, and performed cluster analysis to identify factors affecting multiple-medication use. Results: The PP and PIM use prevalence was $38.4 \%$ and $31.9 \%$, respectively. The median overall survival (OS) for $\mathrm{PP}(+)$ and $\mathrm{PP}(-)$ patients was 19.4 months (95\% CI $=14.1-24.8)$ and 27.3 months $(95 \% \mathrm{CI}=22.6-36.4)$, respectively $(\mathrm{P}<0.001)$. Multivariate analysis revealed a significant correlation between PP and OS. The frequency of unexpected hospitalization during EGFR-TKI treatment was higher in $\mathrm{PP}(+)$ compared to $\mathrm{PP}(-)$ patients $(49.4 \%$ vs. $29.4 \% ; \mathrm{P}=0.0032 ; \mathrm{OR}=2.34 ; 95 \% \mathrm{CI}=1.31-4.23)$. Conclusion: $\mathrm{PP}$ is an independent prognostic factor in older NSCLC patients taking EGFR-TKIs. PP can be used as a simple indicator of such patients' comorbidities and symptoms or as a predictive marker of unexpected hospitalization during treatment.
\end{abstract}

\begin{abstract}
Aim: Polypharmacy (PP) is a common problem among the older adults and has a potential effect on healthrelated problems. However, the significance of PP in older advanced non-small cell lung cancer (NSCLC) patients and those on oral molecular-targeted anticancer agents is unclear.
\end{abstract}

Methods: This retrospective study reviewed the records of 334 advanced NSCLC patients who underwent epidermal growth factor receptor tyrosine kinase inhibitor (EGFR-TKI) treatment. PP was defined as $>=5$ concomitant medications. Potentially inappropriate medication (PIM) use was measured using the updated screening tool of older people's prescriptions (STOPP) ver. 2 criteria. We also estimated survival distributions using the Kaplan-Meier method, compared between-group differences using the log-rank test, explored potential predictors of survival using Cox regression, and performed cluster analysis to identify factors affecting multiple-medication use.

Results: The PP and PIM use prevalence was $38.4 \%$ and $31.9 \%$, respectively. The median overall survival (OS) for $\mathrm{PP}(+)$ and $\mathrm{PP}(-)$ patients was 19.4 months $(95 \% \mathrm{CI}=14.1-24.8)$ and 27.3 months $(95 \% \mathrm{CI}=$ 22.6-36.4), respectively $(P<0.001)$. Multivariate analysis revealed a significant correlation between $\mathrm{PP}$ and OS. The frequency of unexpected hospitalization during EGFR-TKI treatment was higher in $\mathrm{PP}(+)$ compared to $\mathrm{PP}(-)$ patients ( $49.4 \%$ vs. $29.4 \% ; P=0.0032$; $\mathrm{OR}=2.34 ; 95 \% \mathrm{CI}=1.31-4.23)$.

Conclusion: PP is an independent prognostic factor in older NSCLC patients taking EGFR-TKIs. PP can be used as a simple indicator of such patients' comorbidities and symptoms or as a predictive marker of 
unexpected hospitalization during treatment.

\section{Introduction}

Polypharmacy (PP) is a simple summary of a patient's prescription status in terms of the number of drugs concurrently prescribed to him or her. PP is a common problem among older patients, with a potential effect on health-related problems.[1-3] PP can lead to undesirable clinical outcomes, such as adverse drug reactions, drug-drug interactions, reduced adherence to drugs, and excessive health resource use[4]. Potentially inappropriate medication (PIM) use reflects the prescription appropriateness and not just in terms of the numbers of drugs prescribed[5,6]. PIM use is evaluated on the basis of several criteria, such as the screening tool of older people's prescriptions (STOPP), the screening tool to alert to right treatment (START),[7] Beers Criteria, [8] and the medication appropriateness index (MAI) [9].

Older cancer patients can suffer from a higher rate of comorbidity, frailty, and geriatric syndrome, putting them at high risk of PP[10]. Both PP and PIM use are attracting attention in the field of oncology with regard to the general geriatric population[11-15]. Studies have shown the prevalence and effect of PP on chemotherapy outcomes, such as survival and adverse events (AEs), in advanced cancer patients[14, 16-18]. The mortality in lung cancer, which accounts for $20 \%$ of all cancer deaths, is high compared to other cancers. The reason is that few lung cancer cases are diagnosed at the early stage[19, 20], and $~ 60 \%$ of lung cancer patients present with metastasis at diagnosis, which is much higher compared to other common cancers (e.g., $22 \%$ for colorectal and $6 \%$ for breast cancer)[21]. The most common metastatic site is bone, followed by the lungs, brain, adrenal glands, and liver [22]. Distant metastasis along with a primary lesion and its invasion to adjacent structures cause pain and dyspnea. Therefore, multiple medications are often required control symptoms in advanced cancer patients. Studies have shown a higher rate of opioid use among lung cancer patients compared to other cancers[23-25]. In addition, older cancer patients are naturally prone to $\mathrm{PP}$ because of the relatively high prevalence of noncancer multimorbidity with aging. Lung cancer has a median onset age of $\sim 70$ years and is therefore commonly observed in the elderly[26].

However, studies have not clearly described the clinical significance of PP in advanced lung cancer patients and have included only a small number of patients. In addition, data on patients undergoing novel therapy, such as oral molecular-targeted anticancer agents (e.g., epidermal growth factor receptor tyrosine kinase inhibitors [EGFR-TKIs]), are scarce[27]. Also, the applicability of updated STOPP ver. 2 criteria[7] as an assessment tool of PIM use in oncology practice has never been investigated. Therefore, this study investigated the prevalence and effect of PP and PIM use according to STOPP ver. 2 (PIM-STOPP v2) on outcomes for older advanced non-small cell lung cancer (NSCLC) patients treated with EGFR-TKIs.

\section{Methods}

\section{Patients}

This retrospective, nonrandomized study reviewed the electronic medical records (EMRs) of 334 advanced NSCLC patients who underwent EGFR-TKI treatment between 2003 and 2019 at the Tokyo Metropolitan Cancer and Infectious Diseases Center Komagome Hospital, Japan. The inclusion criteria were as follows: (1) age $>=65$ years; (2) histologically or cytologically confirmed unresectable locally advanced (stage III), metastatic (stage IV), or recurrent EGFR -mutated NSCLC; (3) EGFR-TKI (only gefitinib, erlotinib, afatinib, or osimertinib) administration, either as first-line or as later-line therapy; and (4) evaluable for concomitant medications at EGFR-TKI treatment initiation.

We obtained the following baseline characteristics from EMRs: sex; age; body mass index (BMI); smoking status (Brinkman index $=$ daily number of cigarettes $\times$ years); Eastern Cooperative Oncology GroupPerformance Status (ECOG-PS); Charlson Comorbidities Index (CCI), including NSCLC itself; and Gustave Roussy Immune Score (GRIm-Score) calculated from serum albumin levels; lactate dehydrogenase (LDH); neutrophil-to-lymphocyte ratio (NLR) [28]; histological subtype;EGFR mutation status and subtypes; clinical staging (UICC Tumor, Node, Metastasis [TNM] Classification of Malignant Tumors, $8^{\text {th }}$ edition)[29]; number of organs involved in metastasis; lines of EGFR-TKI treatment; and concomitant medications. 


\section{Evaluation of concomitant medications}

A concomitant medication was defined as any therapeutic drug used to manage a comorbid condition in addition to NSCLC between the last visit and day 1 of EGFR-TKI treatment. All medications administered to every patient at EGFR-TKI treatment initiation were reviewed. Oral, injection (e.g., intravenous, intramuscular, and subcutaneous), suppository, and inhalant medications were included, while as-needed and topical medications were excluded. PP was defined as five or more concomitant medications. For compounding agents, we counted each ingredient separately. PIM use was measured using STOPP ver. 2 criteria, [7] a screening tool for detecting potentially inappropriate prescribing in the elderly (age $>=65$ years). In addition to PIM-STOPP v2, we checked PIM use related to EGFR-TKI treatment (PIM-TKI). PIM-TKI was defined as the use of one of the following: (1) concomitant use of cytochrome P450 3A4 (CYP3A4) inhibitors/inducers with gefitinib, erlotinib, or osimertinib[30-32]; (2) concomitant use of CYP1A2 inhibitors with erlotinib[32]; (3) concomitant use of medications affecting the gastric potential of hydrogen $(\mathrm{pH})$, such as antacids with gefitinib or erlotinib[30,32]; and (4) concomitant use of P-glycoprotein (P-gp) inhibitors/inducers with afatinib[33].

\section{Evaluation of undesirable outcomes}

EGFR-TKI-related AEs from EGFR-TKI treatment initiation to the first documented disease progression or death were recorded according to the National Cancer Institute Common Terminology Criteria for Adverse Events (NCI-CTCAE) ver. 4.0. The highest-grade AEs during each therapy were recorded. We also reviewed any unexpected inpatient hospitalizations from EGFR-TKI treatment initiation to the first documented disease progression or death. An unexpected hospitalization event was defined as one of the following: (1) exacerbation of NSCLC, which needed inpatient management despite EGFR-TKI treatment; (2) EGFR-TKIrelated AEs, which needed inpatient management; and (3) complications unrelated to NSCLC or EGFR-TKI treatment, which needed inpatient management.

\section{Statistical analysis}

Descriptive statistics was used to summarize baseline characteristics. Fisher's exact test for categorical data and a Mann-Whitney U test for continuous variables were used to assess between-group differences at the baseline or AE incidence during EGFR-TKI treatment. Progression-free survival (PFS) was defined as the time from EGFR-TKI treatment initiation to the first documented disease progression or death, while overall survival (OS) was defined as the time from EGFR-TKI treatment initiation to the date of death regardless of the cause of death. Patients without disease progression or those who died by the time of analysis were censored at the date of last contact. Survival distributions were estimated using the Kaplan-Meier method, and between-group differences were compared using the log-rank test. The potential predictors of survival were explored using Cox regression. Characteristics with $P<0.05$ after univariate analysis were included in multivariate analysis. Cluster analysis was conducted to identify factors affecting PIM use. CCI scores, number of organs involved in metastasis, the ECOG-PS, and GRIm-Score were standardized on their ranges and used in cluster analysis. In addition, hierarchical cluster analysis with Ward's method was performed with squared Euclidean distances. All $P$-values were two-sided, and $P<0.05$ was considered statistically significant. All statistical analyses were performed using R 3.6.3.

\section{Results}

\section{Baseline characteristics}

Of the total 334 older advanced NSCLC patients, $232(69.5 \%)$ patients (172 females and 60 males) aged $>=65$ years at EGFR-TKI treatment initiation were included in the study (Figure 1 ). Their median age was 73 years (range $=65-88$ years). On the basis of the TNM Classification of Malignant Tumors, $8^{\text {th }}$ edition, 8 (3.4\%), 46 (19.8\%), 121 (52.2\%), and 57 (24.6\%) patients presented with stage III, stage IVA, stage IVB, and recurrent disease, respectively. At EGFR-TKI treatment initiation, $156(67.2 \%)$ patients were administered gefitinib; 38 (16.3\%), erlotinib; 10 (4.3\%), afatinib; and 28 (12.1\%), osimertinib. During the overall clinical course, $66(29.0 \%)$ patients, including those initially treated with first- or second-generation EGFR-TKIs, 
were administrated osimertinib (Table 1 ).

\section{PP and PIM prevalence}

The median concomitant medications were 4 (range $=0-16$; interquartile range $[\mathrm{IQR}]=1-6)$ at EGFR-TKI treatment initiation. The PP prevalence ([?]5 concomitant medications) was 89/232 (38.4\%) (Figure 2 ). According to STOPP ver. 2 criteria, 74 (31.9\%) older advanced NSCLC patients took at least one medication that was deemed PIM. Of these 74 PIM-STOPP v2 $(+)$ patients, $16(21.6 \%)$ violated three or more STOPP ver. 2 criteria checkpoints (Supplementary Table 1 ). According to our criteria, defined in the Materials and Methods section, $66(28.5 \%)$ patients took at least one medication deemed PIM-TKI (Supplementary Table 2$)$. $\mathrm{PP}(+)$ patients were more likely to take at least one PIM-STOPP v2 $(51.7 \%$ vs. $19.6 \% ; P<$ 0.001 ; odds ratio $[\mathrm{OR}]=4.36 ; 95 \%$ confidence interval $[\mathrm{CI}]=2.35-8.25)$ and PIM-TKI $(52.3 \%$ vs. $14.2 \% ; P$ $<0.001 ; \mathrm{OR}=6.56 ; 95 \% \mathrm{CI}=3.38-13.15)$ compared to $\mathrm{PP}(-)$ patients.

\section{Survival analysis in overall older advanced NSCLC patients}

The median follow-up time from EGFR-TKI treatment initiation was 19.4 months. The median PFS of all older advanced NSCLC patients treated with immune checkpoint inhibitors (ICIs) was 12.6 months (95\% CI $=10.7-14.7)$, and the median OS time was 24.5 months $(95 \% \mathrm{CI}=20.3-27.7)$.

Survival analysis in $\mathbf{P P}(+)$ and $\mathrm{PP}(-)$ older advanced NSCLC

\section{patients}

The median PFS of $\mathrm{PP}(+)(n=89)$ and $\mathrm{PP}(-)(n=143)$ older advanced NSCLC patients was 9.8 months $(95 \% \mathrm{CI}=7.8-14.0)$ and 13.8 months $(95 \% \mathrm{CI}=11.5-16.3)$, respectively $(P=0.047)$ (Figure 3A $)$, while the median OS was 19.4 months $(95 \% \mathrm{CI}=14.1-24.8)$ and 27.3 months $(95 \% \mathrm{CI}=22.6-36.4)$, respectively $(P<0.001)$ (Figure 3B ).

\section{Survival analysis in PIM-STOPP v2 (+) and PIM-STOPP v2 (-) older advanced NSCLC} patients

The median PFS of PIM-STOPP v2 $(+)(n=74)$ and PIM-STOPP v2(-) $(n=158)$ older advanced NSCLC patients was 10.1 months $(95 \% \mathrm{CI}=7.8-13.8)$ and 14.3 months $(95 \% \mathrm{CI}=11.4-16.1)$, respectively $(P=$ 0.14) (Supplementary Figure 1A ), while the median OS was 19.8 months $(95 \%$ CI $=14.1-25.9)$ and 26.0 months $(95 \%$ CI $=22.6-33.1)$, respectively $(P=0.06)$ (Supplementary Figure 1B ).

\section{Univariate analysis for the PFS and OS}

Univariate analysis showed that the PFS is significantly correlated with the ECOG-PS, BMI, number of organs involved in metastasis, EGFRmutation status, GRIm-Score, and PP, while the OS is significantly correlated with age, the ECOG-PS, BMI, number of organs involved in metastasis, EGFR mutation status, osimertinib use in the clinical course, GRIm-Score, and PP (Supplementary Table 3 ).

\section{Multivariate analysis for the PFS and OS}

Multivariate analysis showed revealed that the PFS is significantly correlated with the BMI, EGFR mutation status, and GRIm-Score but not with PP, while the OS is significantly correlated with the ECOG-PS, BMI, number of organs involved in metastasis, EGFR mutation status, osimertinib use in the clinical course, GRIm-Score, and PP (Table 2 ).

\section{Undesirable patient outcomes}

During EGFR-TKI treatment, of the total 232 older advanced NSCLC patients, 72 (31.0\%) experienced $>=$ grade 3 EGFR-TKI-related AEs, of which 30 (41.7\%) patients experienced liver function-related AEs (e.g., elevated transaminase), 23 (31.9\%) patients experienced skin-related AEs (e.g., rash, paronychia), and $12(16.7 \%)$ patients experienced gastrointestinal AEs (e.g., diarrhea, nausea, loss of appetite). In addition, 5 patients experienced $>=$ grade 3 pneumonitis, Of the total 232 patients, $86(37.1 \%)$ had at least one unexpected hospitalization and $21(17.2 \%)$ patients had two or more hospitalizations. In addition,40 (17.2\%) 
patients were hospitalized because of exacerbation of NSCLC, 23 (9.9\%) because of EGFR-TKI-related AEs, and $37(16.0 \%)$ because of complications unrelated to NSCLC or EGFR-TKI-related AEs.

\section{Undesirable outcomes in PP(+) and PP(-) older advanced NSCLC patients}

We found no significant difference in the incidence of severe ( $>=$ grade 3) EGFR-TKI-related AEs between $\mathrm{PP}(+)$ and $\mathrm{PP}(-)$ older advanced NSCLC patients $(29.2 \%$ vs. $32.2 \% ; P=0.66 ; \mathrm{OR}=0.87 ; 95 \% \mathrm{CI}=$ 0.47-1.60). Overall unexpected hospitalization during EGFR-TKI treatment was more in $\mathrm{PP}(+)$ patients compared to $\mathrm{PP}(-)$ patients $(49.4 \%$ vs. $29.4 \% ; P=0.0032$; $\mathrm{OR}=2.34 ; 95 \% \mathrm{CI}=1.31-4.23)$. In addition, $\mathrm{PP}(+)$ patients experienced more frequent unexpected hospitalization because of exacerbation of NSCLC $(29.2 \%$ vs. $9.8 \% ; P<0.001 ; \mathrm{OR}=3.78 ; 95 \% \mathrm{CI}=1.76-8.41)$ or complications unrelated to lung cancer or EGFR-TKI-related AEs (23.6\% vs. $11.2 \% ; P=0.016 ; \mathrm{OR}=2.44 ; 95 \% \mathrm{CI}=1.13-5.37)$ compared to $\mathrm{PP}(-)$ patients. In contrast, we found no significant difference in the frequency of unexpected hospitalization because of EGFR-TKI-related AEs between $\mathrm{PP}(+)$ and $\mathrm{PP}(-)$ patients $(5.6 \%$ vs. $12.6 \% ; P=0.11$; OR $=$ $0.42 ; 95 \% \mathrm{CI}=0.12-1.22)($ Table 3 ).

\section{Number of concomitant medications in patients classified by clinical factors}

The number of concomitant medications was significantly higher in patients with a higher CCI score $(>=7$ vs. $<=6 ; P=0.012)$, metastasis in multiple organs $(>=2$ vs. 0 or $1 ; P=0.035)$, poorer PS ( $>=2$ vs. 0 or $1 ; P=0.029)$, and a higher GRIm-Score $(>=2$ vs. 0 or $1 ; P=0.016)$ (Figure $4 \mathbf{A})$. The cluster analysis dendrogram revealed that compared to other factors, CCI scores have a relatively close correlation with the number of concomitant medications(Figure 4B ).

\section{Discussion}

This study assessed the prevalence and clinical significance of PP and PIM use in older advanced NSCLC patients undergoing EGFR-TKI treatment. According to STOPP ver. 2, $40 \%$ and $30 \%$ of patients showed PP and PIM use, respectively, at the baseline. Multivariate analysis showed that PP is independently correlated with the OS and is also correlated with a higher frequency of unexpected hospitalization during EGFR-TKI treatment.

Studies have included only a small numbers of advanced lung cancer patients and have shown relatively high prevalence $(7 \%-80 \%)$ of PP among the patients and its negative impact on clinical outcomes[11, 16, 3437]. However, the heterogeneity of patients' disease states, baseline characteristics, or treatment modalities across target populations or cancer types might impair the applicability of these results to different clinical situations. In addition, studies on the clinical effects of concomitant medications on the outcomes of oral molecular-targeted anticancer agents are scarce[38]. Only one retrospective study on oral molecular-targeted agents for a small number of advanced NSCLC patients $(n=20)$ taking erlotinib is available[27], and the potential utility of PIM use according to updated STOPP ver. 2 criteria among cancer patients has not yet been investigated.

We observed a relatively high prevalence of PP (89/232 patients, 38.4\%) among older advanced NSCLC patients taking EGFR-TKIs. To some extent, our findings are consistent with previous reports on populations with metastatic solid tumors treated with chemotherapy. Because of differences among studies, the median concomitant medications range from four to nine in previous studies[11, 12, 16, 17, 34-37, 39, 40]. It is difficult to determine whether older advanced NSCLC patients take more medications compared to other cancer patients. Simple comparisons with previous studies might be inappropriate because of different medication thresholds or time slots (simultaneous[16, 17] or continuous[4, 41]) used to measure PP. The most commonly used threshold of $\mathrm{PP}$ is $>=5$ concomitant medications, so medications among older advanced cancer patients might indicate the potential utility of other thresholds (excessive polypharmacy[17], major polypharmacy[12]) to detect high-risk patients. With regard to the time slot, we applied simultaneous PP corresponding to the number of concomitant medications taken by patients at EGFR-TKI treatment initiation. Although 
this measure might fail to cover all medications through a period, such as a total clinical course of cancer treatment, simplicity and feasibility are significant for routine use in clinical practice.

Comorbidities in general populations are evaluated using the CCI[42], age-adjusted CCI[43], and Elixhauser Comorbidity Index (ECI)[44]. However, these measures classify advanced cancer patients to the high-risk group only because of their metastatic states. In addition, the burden of symptoms in advanced cancer patients is not reflected. Some scales, such as the Numerical Rating Scale (NRS), assess symptoms, but PP is a more objective indicator for recognizing symptoms or comorbidities as a whole. Therefore, PP can be useful as a simple indicator or an approximate sum of the burden of physical or psychiatric symptoms, along with comorbidities. Our exploratory analysis might provide a novel perspective on the effects of patients' clinical characteristics on PP use. We found significant differences in the number of concomitant medications between groups classified by several factors. In addition, the cluster analysis dendrogram indicated a relatively close correlation between CCI scores and PP compared to other factors. Although PP is an obviously multifactorial problem, the extent of contributing factors should be considered depending on clinical settings or targeted populations.

With regard to survival, there was a significant correlation between the OS and PP in older advanced NSCLC patients. A recent metanalysis of the correlation between PP and survival outcomes for patients on chemotherapy across 11 prospective and retrospective studies[11] showed significant correlations only in 2 studies (1 on ovarian cancer[45] and the other on acute myeloid leukemia),[46] while the remaining 9 studies, including a study on patients taking oral imatinib for chronic myeloid leukemia[38], did not show any correlation between PP and mortality. Our data might suggest that prognostic significance differs according to cancer type and treatment. Recent developments in molecular-targeted anticancer agents have greatly improved prognosis in patients with actionable oncogenic driver mutations. The strength and novelty of our study are not only because it showed a prognostic effect of PP in older advanced NSCLC patients but also because it indicated the clinical significance of PP among older patients who have access to novel, promising anticancer agents. The frequency of overall unexpected hospitalization during EGFR-TKI treatment was greater in $\mathrm{PP}(+)$ patients, indicating that $\mathrm{PP}$ can also be used as a predictive marker of negative cancer or morbidity-related events during EGFR-TKI treatment. Future analysis focusing more on high-risk medications common among cancer patients (e.g. opioid cancer, steroid cancer) might enhance the accuracy of $\mathrm{PP}$ in prognosis stratification and predictability of negative clinical events.

In this study, we found that PIM use is common among older advanced NSCLC patients. Studies on PIM use in elderly cancer patients that apply the Beers Criteria[47] and MAI[34] have shown that the frequency of PIM use ranges from $10 \%$ to $30 \%$. Using the STOPP ver. 2 criteria, our study showed a relatively high frequency of PIM use (74/232 patients, 31.9\%). The most common PIMs were psychoactive medications, such as benzodiazepines (38/74 patients, $51.4 \%)$. In addition, we observed the use of regular opioids without concomitant laxative use $(5 / 74$ patients, $6.8 \%)$. The reasons these medications were deemed PIMs may reflect the characteristic symptoms of cancer to some extent. Aspirin and calcium channel blockers, which are commonly identified as PIMs, were less common according to STOPP ver. 2 criteria. These differences were probably due to differences in the criteria used to judge PIM use, not just specific characteristics of older advanced NSCLC patients. The existing criteria for evaluating PIM use and PP have been developed for general older adults and do not necessarily consider PIM use in the geriatric oncology population. These measures need to be improved for optimal use in oncology settings.

In addition, concomitant medications that potentially have drug-drug interactions with oral moleculartargeted anticancer agents should be considered. Exploratory analysis showed that roughly one-fourth of patients took at least one medication deemed a PIM-TKI. The median OS for PIM-TKI $(+)$ and PIM-TKI(-) patients was 19.8 and 26.0 months, respectively $(P=0.07)$. Although we observed a numerical difference in the median OS in PIM-TKI $(+)$ and PIM-TKI $(-)$ patients, there was no statistical difference. However, future studies need consider the "appropriateness" of concomitant medications on the basis of cancer treatment or oncologic prognosis modalities according to cancer types. In addition, it is necessary to estimate the comprehensive health risk of PP or PIMs, while avoiding the risk of underuse of necessary medications. 
This study had several limitations. First, it was a retrospective, nonrandomized study conducted at a single institution with a relatively small number of patients, so we could not entirely exclude the possibility of unintentional selection bias. Second, over-the-counter, complementary and alternative, or use-as-needed medications were not considered, which might underestimate the need for medical interventions using medications. Third, the medication adherence of socioeconomic aspects of PP was not assessed because of the retrospective nature of the study. Medication adherence could be critical, especially for oral anticancer agents, and therefore is significant. Currently, a real-world observational study investigating factors that make osimertinib less effective by checking medication adherence (CSPOR-LC7, UMIN000038683) is ongoing in Japan. Fourth, our PIM-TKI definition did not consider the timing of antacid administration. Interactions between antacids and some EGFR-TKIs are well recognized, and clinicians could have instructed our cohort about the appropriate timing. However, our data might have some value in alerting clinician for an appropriate intervention in similar populations. Finally, the treatment strategy for advanced $E G F R$-mutant NSCLC has significantly changed, and several choices, including singlet EGFR-TKIs, combination strategies of cytotoxic agents[48], bevacizumab[49], and ICIs[50], are available. Even in singlet EGFR-TKI treatment, the use of osimertinib as either first-line or subsequent line of treatment,[51, 52] is growing. Different drugdrug interactions between treatment modalities must be considered so that truly relevant interactions and applicability in current practice can be determined. Future prospective studies with larger cohorts, reflecting recent updates, are required in order to validate our findings.

In conclusion, PP is a common problem and an independent prognostic factor in older advanced NSCLC patients undergoing EGFR-TKI treatment. PP can be used as a simple indicator of the patients' comorbidities and symptoms or as a predictive marker of unexpected hospitalizations during treatment.

Acknowledgments: We would like to thank Enago (https://www.enago.jp/) for the English language review.

Conflicts of Interest: YH has received personal fees from AstraZeneca, Eli Lilly Japan, Taiho Pharmaceutical, Chugai Pharmaceutical, Ono Pharmaceutical, Bristol-Myers Squibb, Kyowa Kirin, and CSL Behring, outside the submitted work. No other potential conflicts of interest were reported.

Funding information: This study did not receive any specific grant from funding agencies in the public, commercial, or not-for-profit sectors.

Data availability statement: The datasets used and analyzed during the present study are available from the corresponding author on reasonable request.

Ethics approval: The study protocol was approved by the Ethics Committee of the Tokyo Metropolitan Cancer and Infectious Diseases Center Komagome Hospital (approval number: 2352) and conducted in accordance with the tenets of the Declaration of Helsinki.

Authors' contributions: TH, TM and AS conceptualized this study. TH and TM acquired the clinical data. TH, TM, AS, YI and YH were responsible for the interpretation of the data. TH and YH drafted the manuscript. All authors have read and approved the current version of the manuscript.

\section{References}

1. Rankin A, Cadogan CA, Patterson SM, Kerse N, Cardwell CR, Bradley MC, Ryan C, Hughes C: Interventions to improve the appropriate use of polypharmacy for older people. Cochrane Database Syst Rev 2018, 9:CD008165.

2. Gutierrez-Valencia M, Izquierdo M, Cesari M, Casas-Herrero A, Inzitari M, Martinez-Velilla N: The relationship between frailty and polypharmacy in older people: A systematic review. Br J Clin Pharmacol 2018, 84(7):1432-1444.

3. Fried TR, O'Leary J, Towle V, Goldstein MK, Trentalange M, Martin DK: Health outcomes associated with polypharmacy in community-dwelling older adults: a systematic review. J Am Geriatr Soc 2014, 62(12):2261-2272. 
4. Cahir C, Fahey T, Teeling M, Teljeur C, Feely J, Bennett K: Potentially inappropriate prescribing and cost outcomes for older people: a national population study. Br J Clin Pharmacol 2010, 69(5):543-552.

5. Motter FR, Fritzen JS, Hilmer SN, Paniz EV, Paniz VMV: Potentially inappropriate medication in the elderly: a systematic review of validated explicit criteria. Eur J Clin Pharmacol 2018, 74(6):679-700.

6. Storms H, Marquet K, Aertgeerts B, Claes N: Prevalence of inappropriate medication use in residential long-term care facilities for the elderly: A systematic review. Eur J Gen Pract 2017, 23(1):69-77.

7. O'Mahony D, O'Sullivan D, Byrne S, O'Connor MN, Ryan C, Gallagher P: STOPP/START criteria for potentially inappropriate prescribing in older people: version 2. Age Ageing 2015, 44(2):213-218.

8. By the American Geriatrics Society Beers Criteria Update Expert P: American Geriatrics Society 2015 Updated Beers Criteria for Potentially Inappropriate Medication Use in Older Adults. J Am Geriatr Soc2015, 63(11):2227-2246.

9. Hanlon JT, Schmader KE, Samsa GP, Weinberger M, Uttech KM, Lewis IK, Cohen HJ, Feussner JR: A method for assessing drug therapy appropriateness. J Clin Epidemiol 1992, 45(10):1045-1051.

10. Wildiers H, Heeren P, Puts M, Topinkova E, Janssen-Heijnen ML, Extermann M, Falandry C, Artz A, Brain E, Colloca $\mathrm{G}$ et al : International Society of Geriatric Oncology consensus on geriatric assessment in older patients with cancer. J Clin Oncol 2014, 32(24):2595-2603.

11. Mohamed MR, Ramsdale E, Loh KP, Arastu A, Xu H, Obrecht S, Castillo D, Sharma M, Holmes HM, Nightingale G et al : Associations of Polypharmacy and Inappropriate Medications with Adverse Outcomes in Older Adults with Cancer: A Systematic Review and Meta-Analysis. Oncologist 2020, 25(1):e94-e108.

12. Maggiore RJ, Gross CP, Hurria A: Polypharmacy in older adults with cancer. Oncologist 2010, 15(5):507522.

13. Sharma M, Loh KP, Nightingale G, Mohile SG, Holmes HM: Polypharmacy and potentially inappropriate medication use in geriatric oncology.J Geriatr Oncol 2016, 7(5):346-353.

14. LeBlanc TW, McNeil MJ, Kamal AH, Currow DC, Abernethy AP: Polypharmacy in patients with advanced cancer and the role of medication discontinuation. Lancet Oncol 2015, 16(7):e333-341.

15. Lees J, Chan A: Polypharmacy in elderly patients with cancer: clinical implications and management. Lancet Oncol 2011, 12(13):1249-1257.

16. Oldak S, Ioannou S, Kamath P, Huang M, George S, Slomovitz B, Schlumbrecht M: Polypharmacy in Patients with Ovarian Cancer. Oncologist 2019, 24(9):1201-1208.

17. Hong S, Lee JH, Chun EK, Kim KI, Kim JW, Kim SH, Lee YG, Hwang IG, Kim JY, Koh SJ et al : Polypharmacy, Inappropriate Medication Use, and Drug Interactions in Older Korean Patients with Cancer Receiving First-Line Palliative Chemotherapy. Oncologist 2019.

18. Calip GS, Xing S, Jun DH, Lee WJ, Hoskins KF, Ko NY: Polypharmacy and Adherence to Adjuvant Endocrine Therapy for Breast Cancer. J Oncol Pract 2017, 13(5):e451-e462.

19. Matsuda A, Matsuda T, Shibata A, Katanoda K, Sobue T, Nishimoto H, Japan Cancer Surveillance Research G: Cancer incidence and incidence rates in Japan in 2008: a study of 25 population-based cancer registries for the Monitoring of Cancer Incidence in Japan (MCIJ) project.Jpn J Clin Oncol 2014, 44(4):388396.

20. Little AG, Gay EG, Gaspar LE, Stewart AK: National survey of non-small cell lung cancer in the United States: epidemiology, pathology and patterns of care. Lung Cancer 2007, 57(3):253-260.

21. Health USNIo: SEER Cancer Stat Facts. 
22. Tamura T, Kurishima K, Nakazawa K, Kagohashi K, Ishikawa H, Satoh H, Hizawa N: Specific organ metastases and survival in metastatic non-small-cell lung cancer. Mol Clin Oncol 2015, 3(1):217-221.

23. Salz T, Lavery JA, Lipitz-Snyderman AN, Boudreau DM, Moryl N, Gillespie EF, Korenstein D: Trends in Opioid Use Among Older Survivors of Colorectal, Lung, and Breast Cancers. J Clin Oncol 2019, 37(12):10011011.

24. Hasegawa T, Oguri T, Osawa T, Sawa T, Osaga S, Okuyama T, Uchida M, Maeno K, Fukuda S, Nishie $\mathrm{H}$ et al : Opioid Dose and Survival of Patients with Incurable Nonsmall Cell Lung Cancer: A Prospective Cohort Study. J Palliat Med 2018, 21(10):1436-1441.

25. Sutradhar R, Lokku A, Barbera L: Cancer survivorship and opioid prescribing rates: A population-based matched cohort study among individuals with and without a history of cancer. Cancer 2017, 123(21):42864293.

26. Vora N, Reckamp KL: Non-small cell lung cancer in the elderly: defining treatment options. Semin Oncol 2008, 35(6):590-596.

27. Todd A, Williamson S, Husband A, Baqir W, Mahony M: Patients with advanced lung cancer: is there scope to discontinue inappropriate medication? Int J Clin Pharm 2013, 35(2):181-184.

28. Bigot F, Castanon E, Baldini C, Hollebecque A, Carmona A, Postel-Vinay S, Angevin E, Armand JP, Ribrag V, Aspeslagh S et al : Prospective validation of a prognostic score for patients in immunotherapy phase I trials: The Gustave Roussy Immune Score (GRIm-Score). Eur J Cancer 2017, 84:212-218.

29. Rami-Porta R, Bolejack V, Crowley J, Ball D, Kim J, Lyons G, Rice T, Suzuki K, Thomas CF, Jr., Travis WD et al : The IASLC Lung Cancer Staging Project: Proposals for the Revisions of the T Descriptors in the Forthcoming Eighth Edition of the TNM Classification for Lung Cancer.J Thorac Oncol 2015, 10(7):9901003.

30. Feuth T: [The start of 2 levels of competence-from the viewpoint of professional content and organization]. TVZ1992(10):371-375.

31. Vishwanathan K, Dickinson PA, So K, Thomas K, Chen YM, De Castro Carpeno J, Dingemans AC, Kim HR, Kim JH, Krebs MG et al : The effect of itraconazole and rifampicin on the pharmacokinetics of osimertinib. Br J Clin Pharmacol 2018, 84(6):1156-1169.

32. Cohen MH, Johnson JR, Chen YF, Sridhara R, Pazdur R: FDA drug approval summary: erlotinib (Tarceva) tablets. Oncologist 2005, 10(7):461-466.

33. Wind S, Schnell D, Ebner T, Freiwald M, Stopfer P: Clinical Pharmacokinetics and Pharmacodynamics of Afatinib. Clin Pharmacokinet 2017, 56(3):235-250.

34. Maggiore RJ, Dale W, Gross CP, Feng T, Tew WP, Mohile SG, Owusu C, Klepin HD, Lichtman SM, Gajra A et al : Polypharmacy and potentially inappropriate medication use in older adults with cancer undergoing chemotherapy: effect on chemotherapy-related toxicity and hospitalization during treatment. $J$ Am Geriatr Soc 2014, 62(8):1505-1512.

35. Park JW, Roh JL, Lee SW, Kim SB, Choi SH, Nam SY, Kim SY: Effect of polypharmacy and potentially inappropriate medications on treatment and posttreatment courses in elderly patients with head and neck cancer.J Cancer Res Clin Oncol 2016, 142(5):1031-1040.

36. Raijmakers NJ, van Zuylen L, Furst CJ, Beccaro M, Maiorana L, Pilastri P, Rossi C, Flego G, van der Heide A, Costantini M: Variation in medication use in cancer patients at the end of life: a cross-sectional analysis. Support Care Cancer 2013, 21(4):1003-1011.

37. Sasaki T, Fujita K, Sunakawa Y, Ishida H, Yamashita K, Miwa K, Saji S, Kato Y, Sasaki Y: Concomitant polypharmacy is associated with irinotecan-related adverse drug reactions in patients with cancer. Int J Clin Oncol 2013, 18(4):735-742. 
38. Iurlo A, Nobili A, Latagliata R, Bucelli C, Castagnetti F, Breccia M, Abruzzese E, Cattaneo D, Fava C, Ferrero D et al : Imatinib and polypharmacy in very old patients with chronic myeloid leukemia: effects on response rate, toxicity and outcome. Oncotarget 2016, 7(48):80083-80090.

39. Kotlinska-Lemieszek A, Paulsen O, Kaasa S, Klepstad P: Polypharmacy in patients with advanced cancer and pain: a European cross-sectional study of 2282 patients. J Pain Symptom Manage 2014, 48(6):1145-1159.

40. Cashman J, Wright J, Ring A: The treatment of co-morbidities in older patients with metastatic cancer. Support Care Cancer 2010, 18(5):651-655.

41. Bjerrum L, Rosholm JU, Hallas J, Kragstrup J: Methods for estimating the occurrence of polypharmacy by means of a prescription database.Eur J Clin Pharmacol 1997, 53(1):7-11.

42. Charlson ME, Pompei P, Ales KL, MacKenzie CR: A new method of classifying prognostic comorbidity in longitudinal studies: development and validation. J Chronic Dis 1987, 40(5):373-383.

43. Charlson M, Szatrowski TP, Peterson J, Gold J: Validation of a combined comorbidity index. J Clin Epidemiol 1994, 47(11):1245-1251.

44. Elixhauser A, Steiner C, Harris DR, Coffey RM: Comorbidity measures for use with administrative data. Med Care 1998, 36(1):8-27.

45. Freyer G, Geay JF, Touzet S, Provencal J, Weber B, Jacquin JP, Ganem G, Tubiana-Mathieu N, Gisserot O, Pujade-Lauraine E: Comprehensive geriatric assessment predicts tolerance to chemotherapy and survival in elderly patients with advanced ovarian carcinoma: a GINECO study. Ann Oncol 2005, 16(11):1795-1800.

46. Elliot K, Tooze JA, Geller R, Powell BL, Pardee TS, Ritchie E, Kennedy L, Callahan KE, Klepin HD: The prognostic importance of polypharmacy in older adults treated for acute myelogenous leukemia (AML). Leuk Res 2014, 38(10):1184-1190.

47. Page RL, 2nd, Linnebur SA, Bryant LL, Ruscin JM: Inappropriate prescribing in the hospitalized elderly patient: defining the problem, evaluation tools, and possible solutions. Clin Interv Aging 2010, 5:75-87.

48. Hosomi Y, Morita S, Sugawara S, Kato T, Fukuhara T, Gemma A, Takahashi K, Fujita Y, Harada T, Minato K et al : Gefitinib Alone Versus Gefitinib Plus Chemotherapy for Non-Small-Cell Lung Cancer With Mutated Epidermal Growth Factor Receptor: NEJ009 Study. J Clin Oncol 2020, 38(2):115-123.

49. Saito H, Fukuhara T, Furuya N, Watanabe K, Sugawara S, Iwasawa S, Tsunezuka Y, Yamaguchi O, Okada M, Yoshimori K et al : Erlotinib plus bevacizumab versus erlotinib alone in patients with EGFRpositive advanced non-squamous non-small-cell lung cancer (NEJ026): interim analysis of an open-label, randomised, multicentre, phase 3 trial.Lancet Oncol 2019, 20(5):625-635.

50. Socinski MA, Jotte RM, Cappuzzo F, Orlandi F, Stroyakovskiy D, Nogami N, Rodriguez-Abreu D, MoroSibilot D, Thomas CA, Barlesi Fet al : Atezolizumab for First-Line Treatment of Metastatic Nonsquamous NSCLC. N Engl J Med 2018, 378(24):2288-2301.

51. Ramalingam SS, Vansteenkiste J, Planchard D, Cho BC, Gray JE, Ohe Y, Zhou C, Reungwetwattana T, Cheng Y, Chewaskulyong B et al : Overall Survival with Osimertinib in Untreated, EGFR-Mutated Advanced NSCLC. $N$ Engl $J$ Med 2020, 382(1):41-50.

52. Mok TS, Wu YL, Ahn MJ, Garassino MC, Kim HR, Ramalingam SS, Shepherd FA, He Y, Akamatsu H, Theelen WS et al : Osimertinib or Platinum-Pemetrexed in EGFR T790M-Positive Lung Cancer. $N$ Engl $J$ Med 2017, 376(7):629-640.

Table 1. Baseline characteristics of older advanced NSCLC patients analyzed in this study $(n=232)$.

\begin{tabular}{llll}
\hline Characteristics & $\mathrm{PP}(-)(n=143)$ & $\mathrm{PP}(+)(n=89)$ & $\mathrm{P}$ \\
\hline Age group, $n(\%)$ & Age group, $n(\%)$ & Age group, $n(\%)$ & Age group, $n$ (\%)
\end{tabular}




\begin{tabular}{|c|c|c|c|}
\hline Characteristics & $\operatorname{PP}(-)(n=143)$ & $\mathrm{PP}(+)(n=89)$ & $\mathrm{P}$ \\
\hline $65-74$ & $87(60.8)$ & $52(58.4)$ & 0.82 \\
\hline [?] 75 & $56(39.2)$ & $37(41.6)$ & \\
\hline Sex, $n(\%)$ & Sex, $n(\%)$ & Sex, $n(\%)$ & Sex, $n(\%)$ \\
\hline Female & $104(72.7)$ & $68(76.4)$ & 0.64 \\
\hline Male & $39(27.3)$ & $21(23.6)$ & \\
\hline $\begin{array}{l}\text { Smoking status, } n \\
(\%)\end{array}$ & $\begin{array}{l}\text { Smoking status, } n \\
\text { (\%) }\end{array}$ & $\begin{array}{l}\text { Smoking status, } n \\
\text { (\%) }\end{array}$ & $\begin{array}{l}\text { Smoking status, } n \\
(\%)\end{array}$ \\
\hline Brinkman index $<400$ & $108(75.5)$ & $60(67.4)$ & 0.23 \\
\hline Brinkman index [?]400 & $35(24.5)$ & $29(32.6)$ & \\
\hline ECOG-PS, $n(\%)$ & ECOG-PS, $n(\%)$ & ECOG-PS, $n(\%)$ & ECOG-PS, $n(\%)$ \\
\hline $0 / 1$ & $116(81.1)$ & $63(70.8)$ & 0.097 \\
\hline [?] 2 & $27(18.9)$ & $26(29.2)$ & \\
\hline BMI, $n(\%)$ & BMI, $n(\%)$ & BMI, $n(\%)$ & BMI, $n(\%)$ \\
\hline$<18.5$ & $36(25.2)$ & $18(20.2)$ & 0.13 \\
\hline [?] 18.5 & $94(65.7)$ & $68(76.4)$ & \\
\hline $\begin{array}{l}\text { Histological } \\
\text { subtypes, } n(\%)\end{array}$ & $\begin{array}{l}\text { Histological } \\
\text { subtypes, } n(\%)\end{array}$ & $\begin{array}{l}\text { Histological } \\
\text { subtypes, } n(\%)\end{array}$ & $\begin{array}{l}\text { Histological } \\
\text { subtypes, } n(\%)\end{array}$ \\
\hline Adenocarcinoma & $133(93.0)$ & $83(93.3)$ & 1.00 \\
\hline Other & $10(7.0)^{a}$ & $6(6.7)^{\mathrm{b}}$ & \\
\hline Staging, $n(\%)$ & Staging, $n(\%)$ & Staging, $n(\%)$ & Staging, $n(\%)$ \\
\hline III & $7(4.9)$ & $1(1.1)$ & 0.33 \\
\hline IVA & $31(21.7)$ & $15(16.9)$ & \\
\hline IVB & $71(49.7)$ & $50(26.2)$ & \\
\hline Recurrence & $34(23.8)$ & $23(25.8)$ & \\
\hline $\begin{array}{l}\text { Number of organs } \\
\text { involved in } \\
\text { metastasis, } n(\%)\end{array}$ & $\begin{array}{l}\text { Number of organs } \\
\text { involved in } \\
\text { metastasis, } n(\%)\end{array}$ & $\begin{array}{l}\text { Number of organs } \\
\text { involved in } \\
\text { metastasis, } n(\%)\end{array}$ & $\begin{array}{l}\text { Number of organs } \\
\text { involved in } \\
\text { metastasis, } n(\%)\end{array}$ \\
\hline $0 / 1$ & $79(55.2)$ & $40(44.9)$ & 0.16 \\
\hline [?] 2 & $64(44.8)$ & $49(55.1)$ & \\
\hline $\begin{array}{l}\text { Presence of brain } \\
\text { metastasis, } n(\%)\end{array}$ & $\begin{array}{l}\text { Presence of brain } \\
\text { metastasis, } n(\%)\end{array}$ & $\begin{array}{l}\text { Presence of brain } \\
\text { metastasis, } n(\%)\end{array}$ & $\begin{array}{l}\text { Presence of brain } \\
\text { metastasis, } n(\%)\end{array}$ \\
\hline Yes & $40(28.0)$ & $25(28.1)$ & 1.00 \\
\hline $\begin{array}{l}\text { Presence of bone } \\
\text { metastasis, } n(\%)\end{array}$ & $\begin{array}{l}\text { Presence of bone } \\
\text { metastasis, } n(\%)\end{array}$ & $\begin{array}{l}\text { Presence of bone } \\
\text { metastasis, } n(\%)\end{array}$ & $\begin{array}{l}\text { Presence of bone } \\
\text { metastasis, } n(\%)\end{array}$ \\
\hline Yes & $47(32.9)$ & $38(42.7)$ & 0.17 \\
\hline $\begin{array}{l}\text { Presence of liver } \\
\text { metastasis, } n(\%)\end{array}$ & $\begin{array}{l}\text { Presence of liver } \\
\text { metastasis, } n(\%)\end{array}$ & $\begin{array}{l}\text { Presence of liver } \\
\text { metastasis, } n(\%)\end{array}$ & $\begin{array}{l}\text { Presence of liver } \\
\text { metastasis, } n(\%)\end{array}$ \\
\hline Yes & $24(16.8)$ & $17(19.1)$ & 0.79 \\
\hline $\begin{array}{l}E G F R \text { mutation } \\
\text { status, } \boldsymbol{n}(\%)\end{array}$ & $\begin{array}{l}E G F R \text { mutation } \\
\text { status, } \boldsymbol{n}(\%)\end{array}$ & $\begin{array}{l}E G F R \text { mutation } \\
\text { status, } \boldsymbol{n}(\%)\end{array}$ & $\begin{array}{l}E G F R \text { mutation } \\
\text { status, } \boldsymbol{n}(\%)\end{array}$ \\
\hline Exon 19 del & $56(39.2)$ & $36(40.4)$ & 0.88 \\
\hline Exon $21 \mathrm{~L} 858 \mathrm{R}$ & $74(51.7)$ & $46(51.7)$ & \\
\hline Other & $11(8.4)^{\mathrm{c}}$ & $7(7.9)^{\mathrm{d}}$ & \\
\hline Initially chosen & Initially chosen & Initially chosen & Initially chosen \\
\hline EGFR-TKIs, $n$ (\%) & EGFR-TKIs, $n$ (\%) & EGFR-TKIs, $n(\%)$ & EGFR-TKIs, $n$ (\%) \\
\hline Gefitinib & $92(64.3)$ & $64(71.9)$ & 0.16 \\
\hline Erlotinib & $22(15.4)$ & $16(18.0)$ & \\
\hline Afatinib & $9(6.3)$ & $1(1.1)$ & \\
\hline Osimertinib & $20(14.0)$ & $8(9.0)$ & \\
\hline
\end{tabular}




\begin{tabular}{|c|c|c|c|}
\hline Characteristics & $\operatorname{PP}(-)(n=143)$ & $\mathrm{PP}(+)(n=89)$ & $\mathrm{P}$ \\
\hline $\begin{array}{l}\text { Combination with } \\
\text { other antitumor } \\
\text { agents, } n(\%)\end{array}$ & $\begin{array}{l}\text { Combination with } \\
\text { other antitumor } \\
\text { agents, } n(\%)\end{array}$ & $\begin{array}{l}\text { Combination with } \\
\text { other antitumor } \\
\text { agents, } n(\%)\end{array}$ & $\begin{array}{l}\text { Combination with } \\
\text { other antitumor } \\
\text { agents, } n(\%)\end{array}$ \\
\hline Yes & $24(16.8)$ & $7(78.7)$ & 0.081 \\
\hline Lines of & Lines of & Lines of & Lines of \\
\hline EGFR-TKIs use & EGFR-TKIs use & EGFR-TKIs use & EGFR-TKIs use \\
\hline First & $117(81.8)$ & $71(79.8)$ & 0.83 \\
\hline Second or later & $26(18.2)$ & $18(20.2)$ & \\
\hline $\begin{array}{l}\text { Osimertinib use in } \\
\text { clinical course, i (\%) }\end{array}$ & $\begin{array}{l}\text { Osimertinib use in } \\
\text { clinical course, i }(\%)\end{array}$ & $\begin{array}{l}\text { Osimertinib use in } \\
\text { clinical course, i (\%) }\end{array}$ & $\begin{array}{l}\text { Osimertinib use in } \\
\text { clinical course, i (\%) }\end{array}$ \\
\hline Yes & $48(33.6)$ & $18(20.2)$ & 0.041 \\
\hline CCI, $n(\%)$ & CCI, $n(\%)$ & CCI, $n(\%)$ & CCI, $n(\%)$ \\
\hline [?] 6 & $99(69.2)$ & $44(49.4)$ & 0.004 \\
\hline [?] 7 & $54(37.8)$ & $45(50.6)$ & \\
\hline GRIm-Score, $n$ (\%) & GRIm-Score, $n$ (\%) & GRIm-Score, $n$ (\%) & GRIm-Score, $n(\%)$ \\
\hline $0 / 1$ & $121(84.6)$ & $68(76.4)$ & 0.25 \\
\hline $2 / 3$ & $20(14.0)$ & $20(22.5)$ & \\
\hline
\end{tabular}

NSCLC, non-small cell lung cancer; PP, polypharmacy; ECOG-PS, Eastern Cooperative Oncology GroupPerformance Status; BMI, body mass index; EGFR, epidermal growth factor receptor; TKI, tyrosine kinase inhibitor; CCI, Charlson Comorbidities Index; GRIm-Score, Gustave Roussy Immune Score.

a Non-small cell carcinoma, not other specified $(n=8)$, adenosquamous carcinoma $(n=1)$, combined histology composed of adenocarcinoma and small-cell carcinoma $(n=1)$.

b Squamous cell carcinoma $(n=2)$, non-small cell carcinoma, not other specified $(n=1)$, adenosquamous carcinoma $(n=1)$, pleomorphic carcinoma $(n=1)$, poorly differentiated carcinoma $(n=1)$.

${ }^{c}$ Exon 18 G719X $(n=5)$, exon 21 L861Q $(n=2)$, exon 20 insertion $(n=1)$, exon 18 G719X + exon20 S768I, exon 19 del + exon 21 L858R $(n=1)$, exon 20 S768I + exon 21 L858R $(n=1)$.

${ }^{\mathrm{d}}$ Exon 18 G719X $(n=3)$, exon $20 \mathrm{~T} 790 \mathrm{M}+$ exon 21 L858R $(n=2)$, exon 18 G719X + exon 19 deletion $(n=1)$, exon 18 G719X + exon21 L858R $(n=1)$.

Table 2. Multivariate analysis of the PFS and OS.

\begin{tabular}{llll}
\hline Variants for PFS & HR & $\mathbf{9 5 \%}$ CI & P \\
\hline ECOG-PS ([?]2 vs. 0/1) & 1.13 & $0.79-1.61$ & 0.52 \\
BMI (<18.5 vs. [?] 18.5) & 1.78 & $1.28-2.50$ & $<0.001^{*}$ \\
Number of organs involved as metastatic lesions ([?] 2 vs. 0/1) & 1.22 & $0.89-1.68$ & 0.21 \\
EGFR mutation status (Exon 21 L858R vs. Exon 19 del) & 1.05 & $0.77-1.43$ & 0.78 \\
EGFR mutation status (Other vs. Exon 19 del) & 2.59 & $1.51-4.45$ & $<0.001^{*}$ \\
GRIm score (High vs. Low) & 1.94 & $1.27-2.97$ & $0.0023^{*}$ \\
Concomitant medications ([?]5 vs. [?] 4) & 1.34 & $0.99-1.82$ & 0.056 \\
Variants for OS & HR & $\mathbf{9 5 \%}$ CI & P \\
Age group ([?] 75 vs. 65-74) & 1.57 & $1.12-2.21$ & $0.017^{*}$ \\
ECOG-PS ([?]2 vs. 0/1) & 1.54 & $1.06-2.25$ & $0.024^{*}$ \\
BMI (<18.5 vs. [?] 18.5) & 1.86 & $1.29-2.67$ & $<0.001^{*}$ \\
Number of organs involved as metastatic lesions ([?] 2 vs. 0/1) & 1.60 & $1.13-2.26$ & $0.0079^{*}$ \\
EGFR mutation status (Exon 21 L858R vs. Exon 19 del) & 1.13 & $0.79-1.60$ & 0.50
\end{tabular}




\begin{tabular}{llll}
\hline Variants for PFS & HR & $\mathbf{9 5 \%}$ CI & P \\
\hline EGFR mutation status (Other vs. Exon 19 del) & 1.91 & $1.08-3.39$ & $0.026^{*}$ \\
Osimertinib use in clinical course (Yes vs. No) & 0.44 & $0.27-0.71$ & $<0.001^{*}$ \\
GRIm score (High vs. Low) & 2.37 & $1.53-3.67$ & $<0.001^{*}$ \\
Concomitant medications ([?]5 vs. [?] 4) & 1.58 & $1.13-2.21$ & $0.0076^{*}$ \\
\hline
\end{tabular}

$* P<0.05$.

PFS < progression-free survival; OS, overall survival; EGFR, epidermal growth factor receptor; TKI, tyrosine kinase inhibitor; HR, hazard ratio; CI, confidence interval; ECOG-PS, Eastern Cooperative Oncology GroupPerformance Status; BMI, body mass index; GRIm-Score, Gustave Roussy Immune Score.

Table 3. Incidence of undesirable clinical outcomes in $\mathrm{PP}(+)$ and $\mathrm{PP}(-)$ older advanced NSCLC patients.

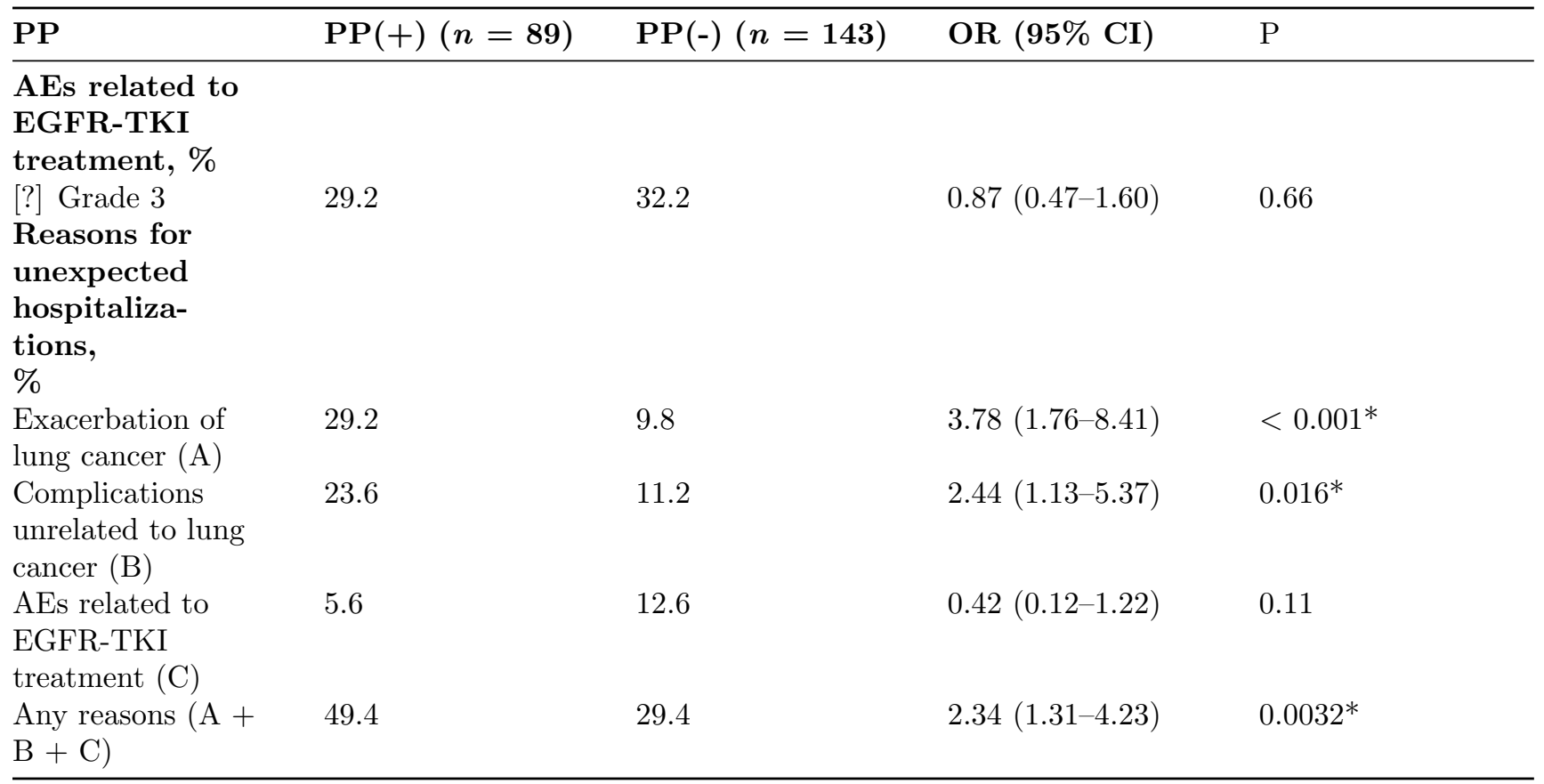

$* P<0.05$.

NSCLS, non-small cell lung cancer; EGFR, epidermal growth factor receptor; TKI, tyrosine kinase inhibitor; PP, polypharmacy; OR: odds ratio; CI, confidence interval; AE, adverse event.

Figure legends

Figure 1. Flow diagram of advanced NSCLC patients enrolled in the study $(n=232)$. NSCLC, non-small cell lung cancer.

Figure 2. Histogram showing the number of concomitant medications and prevalence in older advanced NSCLC patients $(n=232)$. NSCLC, non-small cell lung cancer.

Figure 3. Survival analysis of $\mathrm{PP}(+)$ and $\mathrm{PP}(-)$ older advanced NSCLC patients. Estimated Kaplan-Meier survival curves for (A)PFS and (B) OS for $\mathrm{PP}(+)$ patients $(n=89)$ and $\mathrm{PP}(-)$ patients $(n=143)$. NSCLC, non-small cell lung cancer; PP, polypharmacy; PFS, progression-free survival; OS, overall survival. 
Figure 4. (A) Comparison of number of concomitant medications among older advanced NSCLC patients classified by clinical factors. ${ }^{*} P<0.05$. (B) Cluster analysis dendrogram showing the correlation of CCI scores with the number of concomitant medications. NSCLC, non-small cell lung cancer; $M$ factor, number of organs involved in metastasis; ECOG-PS, Eastern Cooperative Oncology Group-Performance Status; GRIm, Gustave Roussy Immune Score; Total, total number of concomitant medications; CCI, Charlson Comorbidities Index.

Figure 1
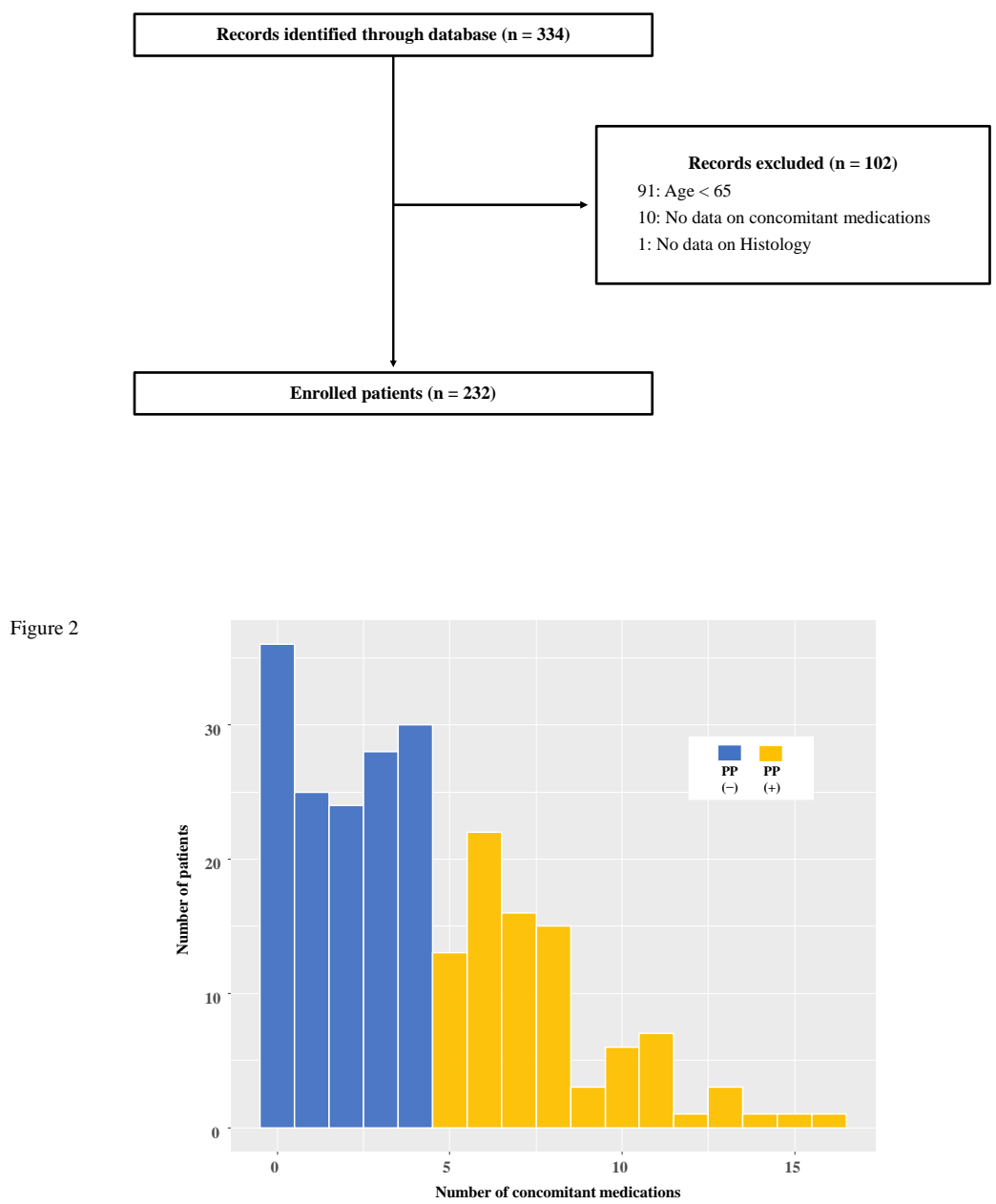
Figure 3
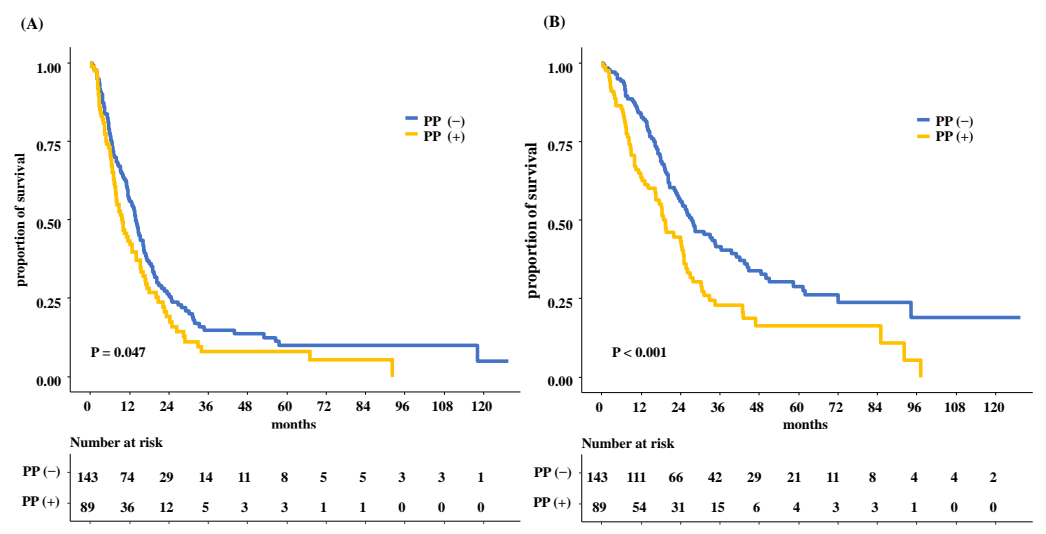

Figure 4

(A)
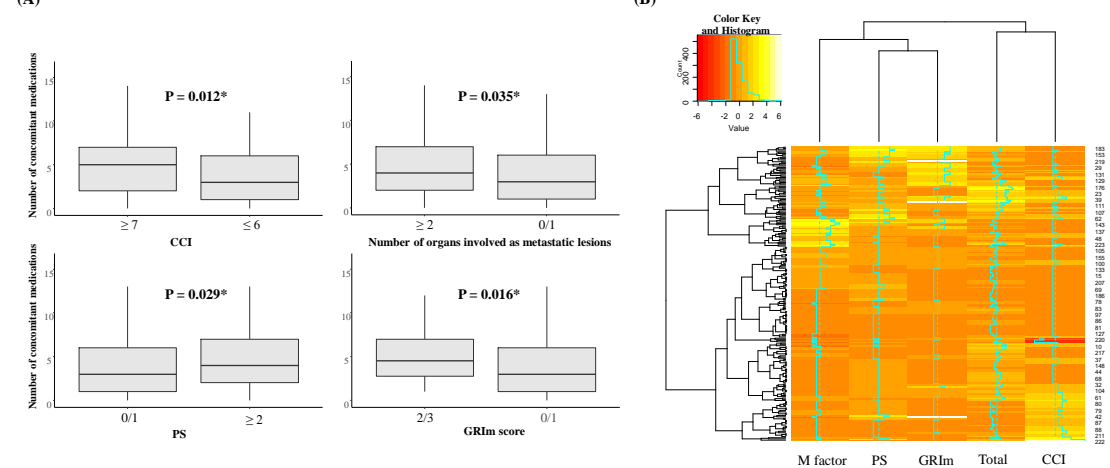

M factor PS GRIm Total CCI

Supplemental figure 1
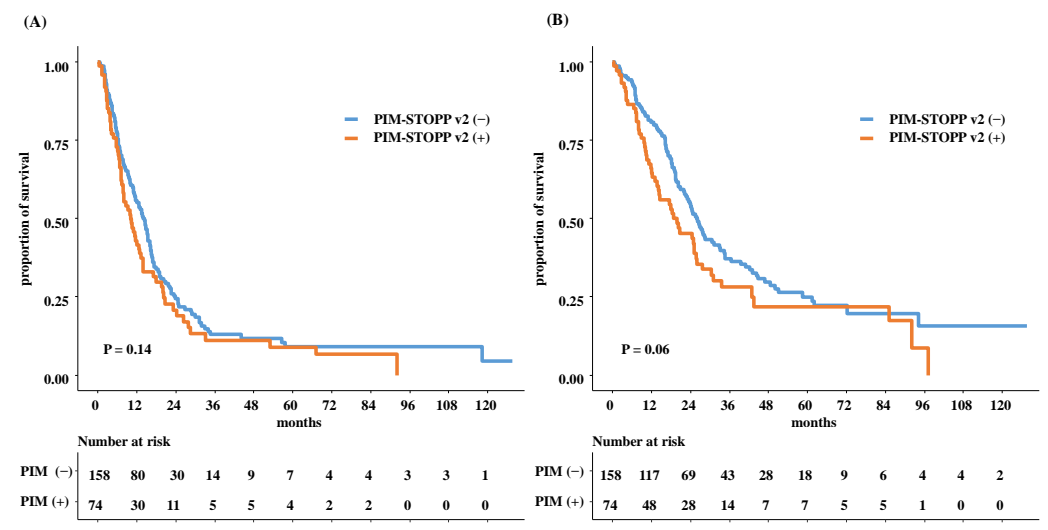